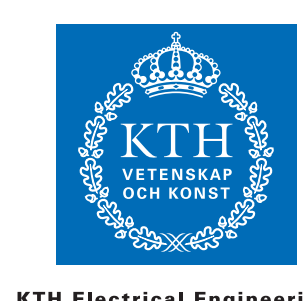

\title{
Polar Codes for Compress-and-Forward in Binary Relay Channels
}

(C)2010 IEEE. Personal use of this material is permitted. However, permission to reprint/republish this material for advertising or promotional purposes or for creating new collective works for resale or redistribution to servers or lists, or to reuse any copyrighted component of this work in other works must be obtained from the IEEE.

RICARDO BLASCO-SERRANO, RAGNAR THOBABEN, VISHWAMBHAR RATHI, AND MIKAEL SKOGLUND

Stockholm 2010

Communication Theory Department

School of Electrical Engineering

KTH Royal Institute of Technology

IR-EE-KT 2010:053 


\title{
Polar Codes for Compress-and-Forward in Binary Relay Channels
}

\author{
Ricardo Blasco-Serrano, Ragnar Thobaben, Vishwambhar Rathi, and Mikael Skoglund \\ School of Electrical Engineering and ACCESS Linnaeus Centre \\ Royal Institute of Technology (KTH). SE-100 44 Stockholm, Sweden \\ Email: \{ricardo.blasco, ragnar.thobaben, vish, mikael.skoglund\}@ee.kth.se
}

\begin{abstract}
We construct polar codes for binary relay channels with orthogonal receiver components. We show that polar codes achieve the cut-set bound when the channels are symmetric and the relay-destination link supports compress-and-forward relaying based on Slepian-Wolf coding. More generally, we show that a particular version of the compress-and-forward rate is achievable using polar codes for Wyner-Ziv coding. In both cases the block error probability can be bounded as $O\left(2^{-N^{\beta}}\right)$ for $0<\beta<\frac{1}{2}$ and sufficiently large block length $N$.
\end{abstract}

\section{INTRODUCTION}

The relay channel characterizes the scenario where a source wants to communicate reliably to a destination with the aid of a third node known as the relay. It was introduced by van der Meulen in [1]. Finding a general expression for its capacity is still an open problem. Some of the most prominent bounds on the capacity were established by Cover and El Gamal in [2]. In particular, they considered the cut-set (upper) bound and proposed the decode-and-forward (DF) and compress-andforward $(\mathrm{CF})$ coding strategies.

Channel polarization and polar codes (PCs), introduced by Arıkan in [3], have emerged as a provable method to achieve some of the fundamental limits of information theory. For example: the capacity of symmetric binary-input discrete memoryless channels (BI-DMC) [3], the symmetric rate-distortion function in source compression with binary alphabets [4], and the entropy of a discrete memoryless source in lossless compression [5], [6]. In their work, Korada and Urbanke also established the suitability of PCs for SlepianWolf [7] and Wyner-Ziv [8] coding in some special cases.

PCs were first used for the relay channel in [9] where they were shown to achieve the capacity of the physically degraded relay channel with orthogonal receivers. The authors showed that the nesting property of PCs for degraded channels reported in [4] allows for DF relaying.

The main contribution of this paper is to show that PCs are also suitable for CF relaying, achieving a particular case of the $\mathrm{CF}$ rate and the cut-set bound in binary symmetric discrete memoryless relay channels with orthogonal receivers. Our approach is based on using constructions of PCs similar to the ones that used in [4] to show the optimality of PCs for the binary versions of the Slepian-Wolf and Wyner-Ziv problems.

This paper is organized as follows. In Section II we review the background, present the scenario, and establish the notation. In Section III we state the main contributions of this paper

This work was supported in part by the European Community's Seventh Framework Programme under grant agreement no 216076 FP7 (SENDORA) and VINNOVA. in the form of two theorems. We prove them in Sections IV and V. The performance of PCs for CF relaying is verified in Section VI using simulations. Section VII concludes our work.

\section{NOTATION, BACKGROUND, AND SCENARIO}

\section{A. Notation}

Random variables (RVs) are represented using capital letters $X$ and realizations using lower case letters $x$. Vectors are represented using bold face $\mathbf{x}$. The $i^{t h}$ component of $\mathbf{x}$ is denoted by $x_{i}$. For a set $F=\left\{f_{0}, \ldots, f_{|F|-1}\right\}$ with cardinality $|F|$ and a vector $\mathbf{x}, \mathbf{x}_{F}$ denotes the subvector $\left(x_{f_{0}}, \ldots, x_{f_{|F|-1}}\right)$. Alphabets are represented with calligraphic letters $\mathcal{X}$.

\section{B. Polar codes for channel and source coding}

Channel polarization is a recently discovered phenomenon based on the repeated application of a simple transformation to $N$ independent identical copies of a basic BI-DMC $W$ to synthesize a set of $N$ (different) polarized BI-DMCs $W^{(i)}$ ( $i \in\{0,1, \ldots, N-1\}$ ) [3]. Let us partition the synthetic channels into three groups. Let the first two contain those channels whose Bhattacharyya parameters $Z\left(W^{(i)}\right)$ are within $\delta$ from 1 and 0 (known as polarized channels), respectively, where $0<\delta<\frac{1}{2}$ is some arbitrarily chosen constant. The third group contains the synthetic channels with Bhattacharyya parameters in $(\delta, 1-\delta)$. For any such $\delta$, as the number of applications of the basic transformation grows, the fraction of synthetic channels in the first and second groups approach $1-I(W)$ and $I(W)$, respectively, where $I(W)$ denotes the symmetric capacity of $W$ (i.e., the mutual information between channel outputs and uniformly distributed inputs). Necessarily, the fraction of channels in the third group vanishes.

It is customary to refer to the group of synthetic channels with Bhattacharyya parameters smaller than $\delta$ as the information set, while the set of channels with parameters greater than $1-\delta$ is usually known as the frozen set. The unpolarized channels are allocated to either of the groups depending on the nature of the problem (e.g., they belong to the frozen set in channel coding and to the information set in source compression). We will denote the frozen set by $F$ and refer to the information set as the complementary of $F$, i.e., $F^{c}$.

Since the Bhattacharyya parameter is an upper bound to the error probability for uncoded transmission, we can construct (symmetric) capacity-achieving PCs as follows [3]. Choose a rate $R<I(W)$ and find the required number of applications of the basic transformation such that the set $F^{c}$ satisfies

$$
R \leq \frac{\left|F^{c}\right|}{N}<I(W)
$$


Use the channels in the information set to transmit the information symbols and send a fixed sequence through the channels in the frozen set. The encoding operation that yields a codeword $\mathbf{x}$ from a vector $\mathbf{u}$ which contains both frozen and information bits ( $\mathbf{u}_{F}$ and $\mathbf{u}_{F^{c}}$ respectively) is linear, i.e., $\mathbf{x}=\mathbf{u} \mathbf{G}_{N}$. After transmission of $\mathbf{x}$ over $W$ a noisy version $\mathbf{y}$ is observed.

In order to decode PCs Arikan [3] proposed a simple successive cancellation (SC) algorithm that estimates the information bits by considering the a posteriori probabilities of the individual synthetic channels $P\left(u_{i} \mid \mathbf{y}_{0}^{N-1}, \hat{\mathbf{u}}_{0}^{i-1}\right)$. The decoder uses its knowledge of the previous frozen bits (i.e., $u_{j}$ for $j<i, j \in F)$ in decoding, thus having to make decisions effectively only on the set of channels with error probability close to 0 . The probability of error for PCs under SC decoding can be bounded as $P_{e} \leq O\left(2^{-N^{\beta}}\right)$ for any $0<\beta<\frac{1}{2}$ provided that the block length $N$ is sufficiently large ${ }^{1}[10]$.

Korada and Urbanke established in [4] that PCs also achieve the symmetric rate-distortion function $R_{s}(D)$ when used for lossy source compression. Their approach was to consider the duality between source and channel coding and employ PCs for channel coding over the test channel that yields $R_{s}(D)$. In this context, the $\mathrm{SC}$ algorithm is used for source compression and the matrix $\mathbf{G}_{N}$ is used for reconstruction.

We reproduce here one result from [4] that will be used later. Consider source compression using PCs when the test channel is a binary symmetric channel with crossover probability $D$ $(\operatorname{BSC}(D))$. Let $\mathbf{E}$ denote the error due to compression using PCs and the SC algorithm as described in [4] and let $P_{\mathbf{E}}(\mathbf{e})$ denote its probability distribution. Let $\mathbf{E}^{\prime}$ denote a vector of independent Bernoulli RVs with $p\left(e^{\prime}=1\right)=D$ and let $P_{\mathbf{E}^{\prime}}\left(\mathbf{e}^{\prime}\right)$ denote its distribution. The optimal coupling between $\mathbf{E}$ and $\mathbf{E}^{\prime}$ is the probability distribution that has marginals equal to $P_{\mathbf{E}}$ and $P_{\mathbf{E}^{\prime}}$ and satisfies $P\left(\mathbf{E} \neq \mathbf{E}^{\prime}\right)=\sum_{\mathbf{x}}\left|P_{\mathbf{E}}(\mathbf{x})-P_{\mathbf{E}^{\prime}}(\mathbf{x})\right|$.

Lemma 1 (Distribution of the quantization error). Let the frozen set $F$ be

$$
F=\left\{i: Z\left(W^{(i)}\right) \geq 1-2 \delta_{N}^{2}\right\} .
$$

for some $\delta_{N}>0$. Then for any choice of the frozen bits $\mathbf{u}_{F}$

$$
P\left(\mathcal{E}_{E}\right)=P\left(\mathbf{E} \neq \mathbf{E}^{\prime}\right) \leq 2|F| \delta_{N} .
$$

This lemma bounds the probability that the error due to compression with $\mathrm{PCs}$ designed upon a $\mathrm{BSC}(D)$ does not behave like a transmission through a $\operatorname{BSC}(D)$.

\section{Relay channel with orthogonal receiver components}

We restrict our attention to the scenario depicted in Fig. 1. It is a particular instance of the relay channel which has orthogonal receiver components [11]. Namely, the probability mass function (pmf) governing the relay channel factorizes as

$$
p\left(y_{d}, y_{s r} \mid x, x_{r}\right)=p\left(y_{s d}, y_{s r} \mid x\right) p\left(y_{r d} \mid x_{r}\right)
$$

with $Y_{D}=\left(Y_{S D}, Y_{R D}\right)$. Moreover, all the alphabets considered here are binary, i.e., $\{0,1\}$. The message to be transmitted by the source is a vector $\mathbf{U}$ that includes both information and

${ }^{1}$ For the sake of brevity we will sometimes omit the phrase "for any $0<$ $\beta<\frac{1}{2}$ provided that the block length $N$ is sufficiently large". frozen bits. This message is encoded into a binary codeword $\mathbf{X}$ which is put into the channel. This gives rise to two observations, one at the relay $\mathbf{Y}_{S R}$ and one at the destination $\mathbf{Y}_{S D}$. Similarly, the relay puts into the channel a vector of bits $\mathbf{X}_{R}$ which leads to the observation $\mathbf{Y}_{R D}$ at the destination. Using $\mathbf{Y}_{R D}$ and $\mathbf{Y}_{S D}$ the relay generates an estimate of the source message $\hat{\mathbf{U}}$.

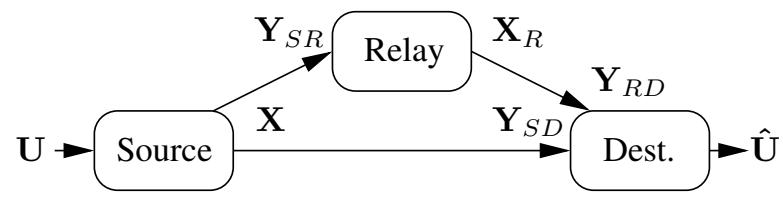

Fig. 1. Relay channel with orthogonal receivers.

The capacity of this simplified model is still unknown. Inner and outer bounds have been established but they are only tight under special circumstances. We consider the following two, which are adapted to our scenario:

Definition 1 (Cut-set upper bound [2]).

$C \leq \max _{p(x) p\left(x_{r}\right)} \min \left\{I\left(X ; Y_{S D}\right)+I\left(X_{R} ; Y_{R D}\right), I\left(X ; Y_{S D}, Y_{S R}\right)\right\}$

Definition 2 (Binary symmetric CF rate [2], [11]).

$$
R_{s}^{C F}=\max _{D} I_{s}\left(X ; Y_{S D}, Y_{Q}\right)
$$

subject to $I_{s}\left(X_{R} ; Y_{R D}\right) \geq I_{s}\left(Y_{Q} ; Y_{S R} \mid Y_{S D}\right)$. Here $Y_{Q}$ is a compressed version of $Y_{S R}$ with the conditional pmf $p\left(y_{q} \mid y_{s r}\right)$ restricted to be equal to that of a $B S C(D)$.

$I_{s}(U ; V)$ denotes the symmetric mutual information between $U$ and $V$. That is, the mutual information when $U$ is uniformly distributed. A similar definition applies to $I_{s}(U ; V \mid T)$.

We follow here the classical scheduling for $\mathrm{CF}$ relaying from [2] that consists of transmitting $m$ messages in $m+1$ time slots, each of which consists of $N$ channel uses. However, for the sake of brevity we will not specify this in the following.

In this paper we assume that the information and frozen bits are drawn i.i.d. according to a uniform distribution. Additionally, we also assume that $p\left(y_{s d}, y_{s r} \mid x\right)$ is such that a uniform distribution on $X$ induces a uniform distribution on $Y_{S R}$. The reason for this is that our constructions rely on interpreting $Y_{S R}$ as the input to a virtual channel ${ }^{2}$.

\section{THE MAIN RESULT}

The main contribution of this paper is to show that sequences of PCs achieve the cut-set bound under some special conditions and the binary symmetric $\mathrm{CF}$ rate, and how to construct them. This is summarized in two theorems:

Theorem 1 (CF relaying with PCs based on Slepian-Wolf coding). For any fixed rate $R<I_{s}\left(X ; Y_{S D}, Y_{S R}\right)$ there exists a sequence of polar codes with block error probability at the destination $P_{e}=\operatorname{Pr}(\hat{\mathbf{U}} \neq \mathbf{U})$ under SC decoding bounded as

$$
P_{e} \leq O\left(2^{-N^{\beta}}\right)
$$

\footnotetext{
${ }^{2}$ Some of the results in this paper can be trivially extended to more general distributions leading to higher rates and/or relaxed constraints without varying the construction of PCs. We omit this due to space limitations.
} 
for any $0<\beta<\frac{1}{2}$ and sufficiently large block length $N$ as long as $I_{s}\left(X_{R} ; Y_{R D}\right) \geq H\left(Y_{S R} \mid Y_{S D}\right)$.

Consider the rates $R_{s}^{C F}$ from Definition 2 with the associated constraint on the (symmetric) capacity of the relaydestination channel.

Theorem 2 (CF relaying with PCs based on Wyner-Ziv coding). For any fixed rate $R<R_{s}^{C F}$ there exists a sequence of PCs with block error probability at the destination $P_{e}=\operatorname{Pr}(\hat{\mathbf{U}} \neq \mathbf{U})$ under $S C$ decoding bounded as

$$
P_{e} \leq O\left(2^{-N^{\beta}}\right)
$$

for any $0<\beta<\frac{1}{2}$ and sufficiently large block length $N$.

\section{COMPRESS-AND-FORWARD RELAYING BASED ON SLEPIAN-WOLF CODING}

Before proceeding with the proof of Theorem 1 we briefly sketch our construction. The idea is to use a sequence of PCs at the source for channel coding that is capacity achieving in the ideal scenario where the destination has access to both $\mathbf{Y}_{S R}$ and $\mathbf{Y}_{S D}$. In order to provide the destination with the relay observation we consider the virtual channel $W_{V}$ that has $\mathbf{Y}_{S R}$ at its input and $\mathbf{Y}_{S D}$ at its output, and conditional pmf

$$
W_{V}\left(y_{s d} \mid y_{s r}\right)=\sum_{x \in \mathcal{X}} p\left(y_{s d}, y_{s r} \mid x\right) \text {. }
$$

If we interpret $\mathbf{Y}_{S R}=\mathbf{v} \mathbf{G}_{N}$ as a codeword from a PC designed for $W_{V}$ (with frozen set $F_{v}$ ), then we only need to transmit the corresponding frozen bits $\left(\mathbf{Y}_{S R} \mathbf{G}_{N}^{-1}\right)_{F_{v}}$ over the relay-destination channel. This will allow the destination to generate the estimate $\hat{\mathbf{Y}}_{S R}$ from $\mathbf{Y}_{S D}$ using the SC algorithm.

Proof of Theorem 1: Design a sequence of PCs for the channel $W: \mathcal{X} \rightarrow \mathcal{Y}_{S D} \times \mathcal{Y}_{S R}$ with transition probabilities given by

$$
W\left(y_{s d}, y_{s r} \mid x\right)=p\left(y_{s d}, y_{s r} \mid x\right) \text {. }
$$

Let $\mathcal{E}, \mathcal{E}_{Y_{S R}}$, and $\mathcal{E}_{R D}$ denote the events $\{\hat{\mathbf{U}} \neq \mathbf{U}\},\left\{\hat{\mathbf{Y}}_{S R} \neq\right.$ $\left.\mathbf{Y}_{S R}\right\}$, and the event of an erroneous relay-destination transmission. Let $\mathcal{E}^{c}, \mathcal{E}_{Y_{S R}}^{c}$, and $\mathcal{E}_{R D}^{c}$ denote their complementary events, respectively. Using this we write

$$
\begin{aligned}
P(\mathcal{E}) & =P\left(\mathcal{E} \mid \mathcal{E}_{R D}\right) P\left(\mathcal{E}_{R D}\right)+P\left(\mathcal{E} \mid \mathcal{E}_{R D}^{c}\right) P\left(\mathcal{E}_{R D}^{c}\right) \\
& \leq P\left(\mathcal{E}_{R D}\right)+P\left(\mathcal{E} \mid \mathcal{E}_{R D}^{c}\right) .
\end{aligned}
$$

If a sequence of PCs is used for the transmission from relay to destination then we know that

$$
P\left(\mathcal{E}_{R D}\right) \leq O\left(2^{-N^{\beta}}\right)
$$

if the transmission rate is below the symmetric capacity of the channel [3]. That is, if

$$
R_{R D}<I_{s}\left(X_{R} ; Y_{R D}\right) .
$$

We now rewrite the term $P\left(\mathcal{E} \mid \mathcal{E}_{R D}^{c}\right)$ in (3) as

$$
\begin{aligned}
P\left(\mathcal{E} \mid \mathcal{E}_{R D}^{c}\right)= & P\left(\mathcal{E} \mid \mathcal{E}_{R D}^{c}, \mathcal{E}_{Y_{S R}}\right) P\left(\mathcal{E}_{Y_{S R}} \mid \mathcal{E}_{R D}^{c}\right) \\
& +P\left(\mathcal{E} \mid \mathcal{E}_{R D}^{c}, \mathcal{E}_{Y_{S R}}^{c}\right) P\left(\mathcal{E}_{Y_{S R}}^{c} \mid \mathcal{E}_{R D}^{c}\right) \\
\leq & P\left(\mathcal{E}_{Y_{S R}} \mid \mathcal{E}_{R D}^{c}\right)+P\left(\mathcal{E} \mid \mathcal{E}_{R D}^{c}, \mathcal{E}_{Y_{S R}}^{c}\right) .
\end{aligned}
$$

The first term in (6) corresponds to the probability of error for PCs used for Slepian-Wolf coding [4]. In order to be able to regenerate $\mathbf{Y}_{S R}$ from $\mathbf{Y}_{S D}$ the destination needs to know the frozen bits $\left(\mathbf{Y}_{S R} \mathbf{G}_{N}^{-1}\right)_{F_{v}}$. Since PCs achieve the symmetric capacity, the size of $F_{v}$ can be bounded as

$$
\frac{\left|F_{v}\right|}{N}>1-I_{s}\left(Y_{S R} ; Y_{S D}\right)=H\left(Y_{S R} \mid Y_{S D}\right)
$$

for sufficiently large $N$. Hence, if the rate used over the relaydestination channel satisfies

$$
R_{R D} \geq H\left(Y_{S R} \mid Y_{S D}\right)
$$

then we can bound the error probability as

$$
P\left(\mathcal{E}_{Y_{S R}} \mid \mathcal{E}_{R D}^{c}\right) \leq O\left(2^{-N^{\beta}}\right) .
$$

The same bound also applies to the second term in (6) since the PC used by the source node for channel coding is designed under the hypothesis that the decoder will have access to $\mathbf{Y}_{S R}$ (expressed by the condition $\mathcal{E}_{Y_{S R}}^{c}$ ), and $\mathbf{Y}_{S D}$. Therefore for any rate $R<I_{s}\left(X ; Y_{S D} Y_{S R}\right)$ we have

$$
P\left(\mathcal{E} \mid \mathcal{E}_{R D}^{c}, \mathcal{E}_{Y_{S R}}^{c}\right) \leq O\left(2^{-N^{\beta}}\right) .
$$

We obtain the desired bound by collecting (4), (8), and (9). The constraint on the symmetric capacity of the relaydestination channel is given by (5) and (7).

Corollary 1. If all the channels are symmetric and $C F$ relaying based on Slepian-Wolf coding is possible, i.e., if $I_{s}\left(X_{R} ; Y_{R D}\right) \geq H\left(Y_{S R} \mid Y_{S D}\right)$, then it achieves the cut-set bound which is given by $I_{s}\left(X ; Y_{S D}, Y_{S R}\right)$.

\section{COMPRESS-AND-FORWARD RELAYING BASED ON WYNER-ZIV CODING}

In the previous section cooperation was implemented by conveying enough information from the relay to the destination so that the latter could reconstruct the observation at the former perfectly. In this section we concentrate on the more relevant case of providing the destination with enough information so that it can get a noisy reconstruction of the relay observation. First we briefly review a construction of nested PCs from [4] and show that it also applies to our scenario. Then we show that by using it, reliable transmission at the binary symmetric $\mathrm{CF}$ rate in (2) is possible.

\section{A. Nested polar codes}

In order to perform $\mathrm{CF}$ relaying for the general case, the relay performs source compression of its observation $\mathbf{Y}_{S R}$ into $\mathbf{Y}_{Q}$ using a $\mathrm{PC}$ constructed (with frozen set $F_{q}$ ) using the $\operatorname{BSC}(D)$ as the test channel, for a given $D$. Therefore, the destination needs to know both the information bits $\mathbf{u}_{F_{q}^{c}}$ and the frozen bits $\mathbf{u}_{F_{q}}$ to reconstruct $\mathbf{Y}_{Q}$. Since the bits $\mathbf{u}_{F_{q}}$ are fixed and known by the relay and the destination, the problem is reduced to providing the destination with the bits in the information set. A straightforward solution is to transmit at rate $R_{R D}=1-h_{b}(D)$ over the relay-destination channel. However, in this way the system does not benefit from the correlation between $Y_{S D}$ and $Y_{S R}$ (and hence $Y_{Q}$ ). 
Assume that the statistical relation $W_{Q}: Y_{S R} \rightarrow Y_{Q}$ is given by a $\operatorname{BSC}(D)$ for a moment. Then it is clear that the virtual channel $W_{V}: Y_{Q} \rightarrow Y_{S R} \rightarrow Y_{S D}$ is degraded with respect to the $\operatorname{BSC}(D)$. A natural tool for this scenario is the construction of nested PCs introduced in [4] for Wyner-Ziv coding. It is based on building one PC for source coding upon $W_{Q}$ and one for channel coding upon $W_{V}$. Nesting comes from the fact that if their respective frozen sets $F_{q}$ and $F_{v}$ are chosen appropriately, then for large enough $N$ we have that $F_{q} \subseteq F_{v}$. That is, all the frozen bits used for source coding have the same value in channel coding over $W_{V}$. This allows the destination to recover $\mathbf{Y}_{Q}$ from the observation $\mathbf{Y}_{S D}$ provided that the rate used for transmission from relay to destination satisfies

$$
\begin{aligned}
R_{R D}=\frac{\left|F_{q}^{c}\right|-\left|F_{v}^{c}\right|}{N} & >I\left(W_{Q}\right)-I\left(W_{V}\right) \\
& =I_{s}\left(Y_{Q} ; Y_{S R} \mid Y_{S D}\right)
\end{aligned}
$$

and that $N$ is sufficiently large [4].

The analysis of the probability of error is similar to that in the proof of Wyner-Ziv coding with PCs. However, here one needs to consider not only the possible errors due to modeling the compression error as a $\operatorname{BSC}(D)\left(\right.$ event $\left.\mathcal{E}_{E}\right)$, but also the errors due to incorrectly decoded relay-destination transmissions (event $\mathcal{E}_{R D}$ ). Let $\mathcal{E}_{Y_{Q}}$ denote the event that the estimate of $\mathbf{Y}_{Q}$ at the destination is wrong. Then we have that

$$
\begin{aligned}
P\left(\mathcal{E}_{Y_{Q}}\right)= & P\left(\mathcal{E}_{Y_{Q}} \mid \mathcal{E}_{E}^{c}\right) P\left(\mathcal{E}_{E}^{c}\right)+P\left(\mathcal{E}_{Y_{Q}} \mid \mathcal{E}_{E}\right) P\left(\mathcal{E}_{E}\right) \\
\leq & P\left(\mathcal{E}_{Y_{Q}} \mid \mathcal{E}_{E}^{c}, \mathcal{E}_{R D}^{c}\right) P\left(\mathcal{E}_{R D}^{c} \mid \mathcal{E}_{E}^{c}\right) \\
& +P\left(\mathcal{E}_{Y_{Q}} \mid \mathcal{E}_{E}^{c}, \mathcal{E}_{R D}\right) P\left(\mathcal{E}_{R D} \mid \mathcal{E}_{E}^{c}\right)+P\left(\mathcal{E}_{E}\right) \\
\leq & P\left(\mathcal{E}_{Y_{Q}} \mid \mathcal{E}_{E}^{c}, \mathcal{E}_{R D}^{c}\right)+P\left(\mathcal{E}_{R D}\right)+P\left(\mathcal{E}_{E}\right) \\
\leq & O\left(2^{-N^{\beta}}\right) .
\end{aligned}
$$

In obtaining (11) we have used the independence of $\mathcal{E}_{E}$ and $\mathcal{E}_{R D}$. All three terms in (11) can be bounded individually as in (12). If (10) is satisfied, the conditions in the first term in (11) guarantee that the nested PC is working under the design hypothesis. The second term follows the bound in (12) if PCs are used for transmission from relay to destination as long as (5) holds. The bound on the last term is due to Lemma 1.

\section{B. Proof of Theorem 2}

Again, we briefly sketch our solution before proceeding with the proof. In this case the channel code used by the source is designed under the assumption that the destination will have access not only to $\mathbf{Y}_{S D}$ but also to $\tilde{\mathbf{Y}}_{Q}$ which results from the concatenation of the source-relay channel and the BSC resulting from the optimization problem in Def. 2. In reality the relay will generate $\mathbf{Y}_{Q}$ using the $\mathrm{SC}$ algorithm and make it available to the destination using the nested PC structure from Section V-A (with the parameter $D$ equal to the one that maximizes (2)). That is, the relay will send to the destination part of the frozen bits that are needed to recover $\mathbf{Y}_{Q}$ from $\mathbf{Y}_{S D}$. Moreover, as the block length increases, the error due to our design based on $\tilde{\mathbf{Y}}_{Q}$ instead of $\mathbf{Y}_{Q}$ will vanish.

Proof of Theorem 2: Choose a transmission rate $R<I_{s}\left(X ; Y_{S D}, Y_{Q}\right)$ and design a sequence of PCs for the channel $W: \mathcal{X} \rightarrow \mathcal{Y}_{S D} \times \mathcal{Y}_{Q}$ with transition probabilities

$$
W\left(y_{s d}, y_{q} \mid x\right)=\sum_{y_{s r} \in \mathcal{Y}_{S R}} W_{Q}\left(y_{q} \mid y_{s r}\right) p\left(y_{s d}, y_{s r} \mid x\right)
$$

where $W_{Q}$ is the $\operatorname{BSC}(D)$ obtained in the maximization in (2) and $p\left(y_{s d}, y_{s r} \mid x\right)$ comes from the channel pmf (1).

Let $\mathcal{E}$ denote the event $\{\hat{\mathbf{U}} \neq \mathbf{U}\}$, and $\mathcal{E}_{Y_{Q}}, \mathcal{E}_{R D}$, and $\mathcal{E}_{E}$ be defined as in Section V-A. Again, $\mathcal{E}^{c}, \mathcal{E}_{Y_{Q}}^{c}, \mathcal{E}_{R D}^{c}$, and $\mathcal{E}_{E}^{c}$ denote their complementary events. Using this we write

$$
\begin{aligned}
P(\mathcal{E}) & =P\left(\mathcal{E} \mid \mathcal{E}_{R D}\right) P\left(\mathcal{E}_{R D}\right)+P\left(\mathcal{E} \mid \mathcal{E}_{R D}^{c}\right) P\left(\mathcal{E}_{R D}^{c}\right) \\
& \leq P\left(\mathcal{E}_{R D}\right)+P\left(\mathcal{E} \mid \mathcal{E}_{R D}^{c}\right) .
\end{aligned}
$$

Again, the bound and the constraint expressed in (4) and (5) respectively apply to the first term in (13) if a sequence of PCs is used for the relay-destination transmission. We now rewrite the last term in (13) as

$$
\begin{aligned}
P\left(\mathcal{E} \mid \mathcal{E}_{R D}^{c}\right)= & P\left(\mathcal{E} \mid \mathcal{E}_{R D}^{c}, \mathcal{E}_{E}\right) P\left(\mathcal{E}_{E} \mid \mathcal{E}_{R D}^{c}\right) \\
& +P\left(\mathcal{E} \mid \mathcal{E}_{R D}^{c}, \mathcal{E}_{E}^{c}\right) P\left(\mathcal{E}_{E}^{c} \mid \mathcal{E}_{R D}^{c}\right) \\
\leq & P\left(\mathcal{E}_{E} \mid \mathcal{E}_{R D}^{c}\right)+P\left(\mathcal{E} \mid \mathcal{E}_{R D}^{c}, \mathcal{E}_{E}^{c}\right) \\
= & P\left(\mathcal{E}_{E}\right)+P\left(\mathcal{E} \mid \mathcal{E}_{R D}^{c}, \mathcal{E}_{E}^{c}\right)
\end{aligned}
$$

where the last step is due to the independence of $\mathcal{E}_{E}$ and $\mathcal{E}_{R D}$. From Lemma 1 we know that

$$
P\left(\mathcal{E}_{E}\right) \leq O\left(2^{-N^{\beta}}\right) .
$$

Finally, we bound the last term in (14) as

$$
\begin{aligned}
P\left(\mathcal{E} \mid \mathcal{E}_{R D}^{c}, \mathcal{E}_{E}^{c}\right)=P\left(\mathcal{E} \mid \mathcal{E}_{R D}^{c}, \mathcal{E}_{E}^{c}, \mathcal{E}_{Y_{Q}}\right) P\left(\mathcal{E}_{Y_{Q}} \mid \mathcal{E}_{R D}^{c}, \mathcal{E}_{E}^{c}\right) \\
\quad+P\left(\mathcal{E} \mid \mathcal{E}_{R D}^{c}, \mathcal{E}_{E}^{c}, \mathcal{E}_{Y_{Q}}^{c}\right) P\left(\mathcal{E}_{Y_{Q}}^{c} \mid \mathcal{E}_{R D}^{c}, \mathcal{E}_{E}^{c}\right) \\
\leq P\left(\mathcal{E}_{Y_{Q}} \mid \mathcal{E}_{R D}^{c}, \mathcal{E}_{E}^{c}\right)+P\left(\mathcal{E} \mid \mathcal{E}_{R D}^{c}, \mathcal{E}_{E}^{c}, \mathcal{E}_{Y_{Q}}^{c}\right) \\
\leq \\
\leq
\end{aligned}
$$

The first term in (16) was already bounded in Section V-A under the constraint in (10), where $D$ is now chosen as the result of the maximization in (2). Bounding the second term is straightforward for the PC used for channel coding at the source relies on the assumptions that $\mathcal{E}_{E}^{c}$ and $\mathcal{E}_{Y_{Q}}^{c}$ hold.

Combining (4), (15), and (17) we obtain the desired bound on $P_{e}$. The constraint comes from (5) and (10).

\section{Simulations}

In this section we present simulation results and comment on the performance for finite block length in the two scenarios: CF based on Slepian-Wolf and on Wyner-Ziv coding. In both cases we have modeled the source-relay and sourcedestination channels as two independent BSCs. The relaydestination link is modeled as a capacity-limited error-free channel. This allows us to concentrate on the performance of the more interesting elements of the system.

The first scenario corresponds to CF based on Slepian-Wolf coding. The crossover probabilities of the source-relay and source-destination BSCs are 0.05 and 0.1 respectively. Accordingly, the bounds in Theorem 1 are $I_{s}\left(X ; Y_{S D}, Y_{S R}\right) \approx 0.83$ and $H\left(Y_{S R} \mid Y_{S D}\right) \approx 0.58$. A non-cooperative strategy would be limited by $I_{s}\left(X ; Y_{S D}\right) \approx 0.53$. 
The behavior of the bit error rate (BER) $\operatorname{Pr}(\hat{U} \neq U)$ is shown in Fig. 2 for different values of three parameters: source transmission rate $(R$, coordinate axis), block length ( $N=2^{n}$, specified by the line marker), and rate over the relay-destination channel ( $R_{R D}$, specified by the line face).

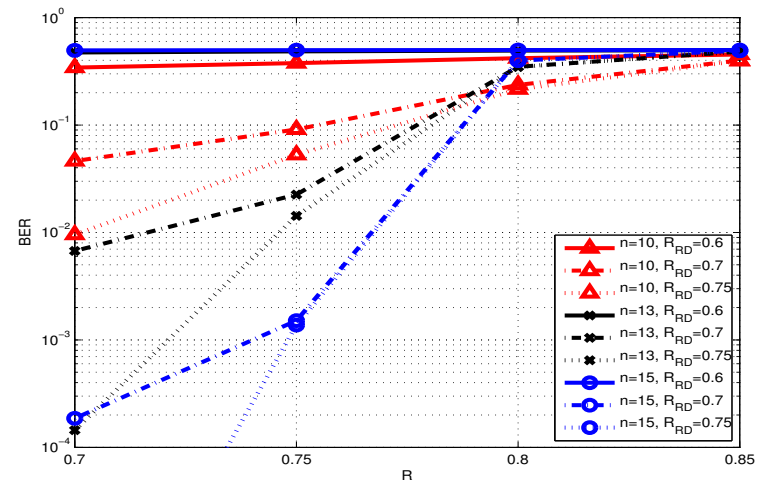

Fig. 2. Performance of CF relaying with PCs based on Slepian-Wolf coding.

As expected the BER is reduced by increasing $n$ for fixed $R$ and $R_{R D}$. Similarly to other coding methods such as Turbo or LDPC codes we observe the appearance of threshold effects around $R \approx 0.8<0.83$ and $R_{R D} \approx 0.65>0.58$. It is expected that with larger blocks their positions will shift towards $I_{s}\left(X ; Y_{S D}, Y_{S R}\right)$ and $H\left(Y_{S R} \mid Y_{S D}\right)$, respectively. For fixed $n$ the BER can be reduced by increasing both gaps to the bounds, i.e., by reducing $R$ and increasing $R_{R D}$. That is, by lowering the efficiency of the system in terms of rate we can improve the BER behavior without adding complexity or delay. However, we observe a saturation effect if only one of the rates is changed. For example, for $R<0.75$ and fixed $R_{R D}$ the BER curves flatten out. This is due to the fact that errors on the channel code over the virtual channel (event $\mathcal{E}_{Y_{S R}}$ ) start dominating the error probability (first term in (6)). A similar effect is observed if only $R_{R D}$ is increased. In this case, the the virtual channel becomes nearly error-free and the error probability is dominated by the weakness of the channel code used by the source.

The second scenario corresponds to $\mathrm{CF}$ relaying based on Wyner-Ziv coding. The crossover probabilities of the source-relay and source-destination BSCs are 0.1 and 0.05 respectively. That is, the relay has an observation of worse quality than that of the destination. In this scenario the relay employs a PC for source compression at a rate of $R_{Q}=0.8$ bits per observation. The limits in Theorem 2 are $I_{s}\left(X ; Y_{S D}, Y_{Q}\right) \approx 0.81, I_{s}\left(Y_{Q} ; Y_{S R} \mid Y_{S D}\right) \approx 0.44$. Without cooperation the scenario is limited by $I_{s}\left(X ; Y_{S D}\right) \approx 0.71$.

The response of the system to the variations in the same parameters as before is shown in Fig. 3. In general the effect is the same as for the Slepian-Wolf case. However, for similar gaps to the different bounds the Wyner-Ziv scenario performs worse than the Slepian-Wolf case. The reason for this is that our construction based on Wyner-Ziv coding contains one more PC that the one based on Slepian-Wolf coding. Moreover, the assumption on the distribution of the compression error is only accurate in the asymptotic regime. Thats is, the addition of the rate-distortion component adds suboptimalities for limited $n$ not only due to its practical implementation (PC), but also due to the modeling of the compression error.

As a final remark we would like to note that even though asymptotically optimal, the performance for small blocks is far away from the bounds. This problem is common to PCs in general [3], [4] and is particularly visible in our case due to the aforementioned idealizations of the system.

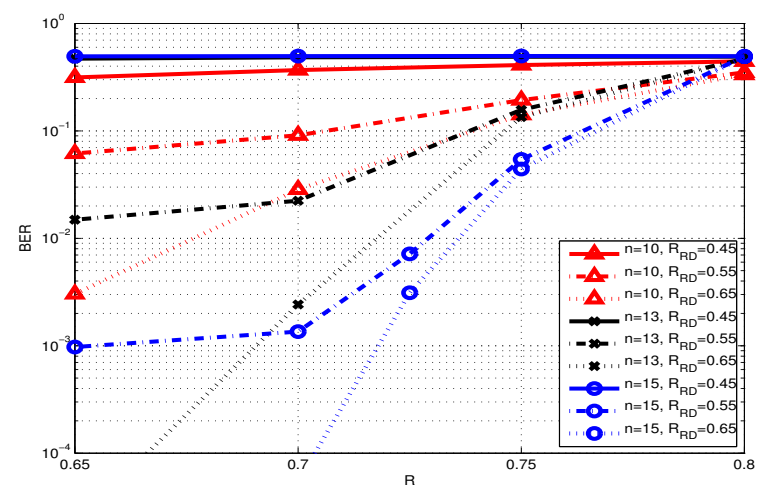

Fig. 3. Performance of CF relaying with PCs based on Wyner-Ziv coding.

\section{CONCLUSION}

We have shown that PCs are suitable for CF relaying in binary relay channels with orthogonal receivers. If all channels are symmetric and the capacity of the relay-destination channel is large enough, CF based on Slepian-Wolf coding achieves the cut-set bound. More generally, for arbitrary capacities of the relay-destination channel transmission at a constrained version of the CF rate is possible by nesting PCs for channel coding into PCs for source coding as in the Wyner-Ziv problem. Our simulation results match the behavior predicted by the theoretical derivations. However, even though asymptotically optimal, the performance for finite block lengths is far away from the limits.

\section{REFERENCES}

[1] E. C. van der Meulen, "Three-terminal communication channels," Advances in Applied Probability, no. 3, pp. 120 - 154, 1971.

[2] T. M. Cover and A. A. El Gamal, "Capacity theorems for the relay channel," IEEE Trans. Inf. Theory, vol. 25, pp. 572 - 584, Sep. 1979.

[3] E. Arıkan, "Channel polarization: A method for constructing capacityachieving codes for symmetric binary-input memoryless channels," IEEE Trans. Inf. Theory, vol. 55, no. 7, pp. 3051-3073, July 2009.

[4] S. B. Korada and R. L. Urbanke, "Polar codes are optimal for lossy source coding," IEEE Trans. Inf. Theory, pp. 1751 -1768, Apr. 2010.

[5] N. Hussami, S. Korada, and R. Urbanke, "Performance of polar codes for channel and source coding," in Proc. IEEE Int. Symp. Inf. Theory, June 2009, pp. 1488-1492.

[6] E. Arikan, "Source polarization," in Proc. IEEE Int. Symp. Inf. Theory, June 2010, pp. 899-903.

[7] D. Slepian and J. Wolf, "Noiseless coding of correlated information sources," IEEE Trans. Inf. Theory, vol. 19, no. 4, July 1973.

[8] A. Wyner and J. Ziv, "The rate-distortion function for source coding with side information at the decoder," IEEE Tran. Inf. Theory, Jan. 1976.

[9] M. Andersson, V. Rathi, R. Thobaben, J. Kliewer, and M. Skoglund, "Nested polar codes for wiretap and relay channels," IEEE Comm. Letters, vol. 14, no. 8, pp. $752-754$, Aug. 2010

[10] E. Arikan and E. Telatar, "On the rate of channel polarization," in Proc. IEEE Int. Symp. Inf. Theory, June 2009, pp. 1493-1495.

[11] Y. H. Kim, "Coding techniques for primitive relay channels," in Proc. in 45 Annual Allerton Conf. Commun., Contr. Comput., Sep. 2007. 